\title{
Generating libraries of iTol2-end insertions at BAC ends using loxP and lox511 Tn10 transposons
}

\author{
Leighcraft A Shakes ${ }^{1}$, Gembu Abe ${ }^{2}$, Mugtaba A Eltayeb ${ }^{1}$, Hope M Wolf ${ }^{1,3}$, Koichi Kawakami ${ }^{2}$ and \\ Pradeep K Chatterjee ${ }^{1 *}$
}

\begin{abstract}
Background: Bacterial Artificial Chromosomes (BACs) have been widely used as transgenes in vertebrate model systems such as mice and zebrafish, for a variety of studies. BAC transgenesis has been a powerful tool to study the function of the genome, and gene regulation by distal cis-regulatory elements. Recently, BAC transgenesis in both mice and zebrafish was further facilitated by development of the transposon-mediated method using the Tol2 element. Tol2 ends, in the inverted orientation and flanking a $1 \mathrm{~kb}$ spacer DNA (iTol2), were introduced into the BAC DNA within the bacterial host using recombination of homologous sequences. Here we describe experiments designed to determine if a simpler and more flexible system could modify BACs so that they would be suitable for transgenesis into zebrafish or mouse embryos using the Tol2 transposase.
\end{abstract}

Results: A new technique was developed to introduce recognition sequences for the Tol2 transposase into BACs in E. coli using the Tn10 transposon vector system. We constructed pTnloxP-iTol2kan and pTnlox511-iTol2kan to introduce the loxP or lox511 site and iTol2 cassette, containing the Tol2 cis-sequences in the inverted orientation, into BACs that have loxP and lox511 sites flanking genomic DNA inserts by Tn10-mediated transposition. The procedure enables rapid generation of a large collection of BACs ready for transgenesis with the iTol2 cassette at the new end of a progressively truncated genomic insert via lox-Cre recombination. The iTol2 ends are efficiently recognized by the Tol2 transposase, and the BACs readily integrate into zebrafish chromosomes.

Conclusion: The new technology described here can rapidly introduce iTol2 ends at a BAC end of choice, and simultaneously generate a large collection of BACs with progressive deletions of the genomic DNA from that end in a single experiment. This procedure should be applicable to a wider variety of BACs containing lox sites flanking the genomic DNA insert, including those with sequence repeats. The libraries of iTol2 inserted BACs with truncations from an end should facilitate studies on the impact of distal cis-regulatory sequences on gene function, as well as standard BAC transgenesis with precisely trimmed genes in zebrafish or mouse embryos using Tol2 transposition.

\section{Background}

The Tol2 transposon system has been used extensively to deliver exogenous DNA into the germline of zebrafish [1-3], and more recently for successfully integrating BAC DNA into the germline of both zebrafish and mice [4]. In this method the iTol2 cassette, comprising of the minimal cis-sequences of Tol2 in an inverted orientation separated by a $\sim 1 \mathrm{~kb}$ spacer, was introduced into a BAC

\footnotetext{
* Correspondence: pchatterjee@nccu.edu

'Julius L. Chambers Biomedical/Biotechnology Research Institute \& Department of Chemistry, North Carolina Central University, 1801 Fayetteville Street, Durham, NC 27707, USA

Full list of author information is available at the end of the article
}

using recombination of homologous sequences within the genomic insert DNA in the bacterial host [5].

A different strategy to modify genomic DNA cloned in BACs uses Cre-lox recombination. This method uses the bacterial Tn10 transposon to introduce exogenous DNA, including lox sites, at random locations in the BAC [6,7]. The inserted lox sites can in turn be recombined with those endogenous to the BAC vector with Cre protein to progressively delete insert DNA from either end efficiently and selectively [8]. The procedure has been used previously to deliver reporter genes and other exogenous DNA cassettes such as sequencing primer sites, mammalian cell-selectable antibiotic resistance genes and EGFP

\section{Biomed Central}


enhancer-traps precisely at the newly created ends of the DNA insert in BACs [7-9]. It is significant that the recombinases involved in this approach, namely Tn10transposase and Cre protein, do not act upon sequence repeats and/or other recombinogenic sites in the genomic DNA insert to rearrange it. This particular characteristic makes the approach applicable to a wider variety of BACs in the public domain, including those with repeated sequences (see reference [10], for example). Here we describe methodology to efficiently place iTol2 cassettes precisely at the ends of the genomic DNA insert using either a $l o x P$ or a $l o x 511$ recombination system. Cre-recombination of the endogenous loxP or lox 511 sites, a constituent of all BACs in the public domain, with the ones inserted by these iTol2-Tn10 transposons simultaneously truncates the DNA from the respective end and delivers the iTol 2 cassette. Large numbers of BACs progressively truncated from either end, with iTol2 placed at the newly created end, can thus be obtained in a single experiment.

\section{Methods}

Construction of Tn10 mini-transposons with iTol2kan cassettes in front of either loxP or lox511

The iTol2-kan DNA cassette was inserted in front of the loxP or lox511 site in the previously described Tn10 transposon plasmids pTnMarkerless2 [11], and pTnlox511(B) markerless1 [8]. The oligonucleotides d (GGCGCGCC TGCTCGAGCCGGGCCCAAGTGATCTC) and d (GGC GCGCCTCTAGATCAGATCTAATACTC) were used to amplify the iTol2-kan DNA cassette. The 1371 bp PCR product was flanked by Asc I sites, and contained the gene for kanamycin resistance which was in turn flanked by inverted $200 \mathrm{bp}$ Tol2R and inverted $150 \mathrm{bp}$ Tol2L [4]. This PCR product was inserted into AT-cloning vector pCR2.1. The DNA isolated from the clones was digested with Asc I enzyme, and the purified DNA fragment inserted at the Asc I sites of plasmids pTnMarkerless2, and pTnlox511(B)markerless1. Both orientations of the iTol2-kan insert in each of the plasmids pTnMarkerless2 and pTnlox511(B)markerless1 were isolated. The mixture of plasmids with both orientations of iTol2kan will be designated as pTnloxP-iTol2kan and pTnlox511-iTol2kan.

\section{Functionalizing BACs with EGFP gene cassettes}

BACs were functionalized with the EGFP gene in one of two ways: using EGFP enhancer-traps in BAC clone $\mathrm{CH} 211-43 \mathrm{O} 16$ containing zebrafish $\mathrm{APPb}$ gene [9], or by galK-mediated recombination in bacteria for BAC clone CH211-163F17 carrying the zebrafish fgf 24 gene. The fgf24 BAC was isolated in the host SW 102 after being functionalized with the EGFP gene using the latter procedure $[4,5]$. Because this host contains a temperature sensitive $\lambda$-prophage, the fgf24:EGFP BAC DNA was isolated and re-introduced by electroporation into the DH10B host.

\section{Generating BAC deletion libraries with pTn/oxP-iTol2kan and pTnlox511-iTol2kan}

Deletion libraries of EGFP-functionalized BACs were made with the iTol2kan-Tn10 transposons as previously described for other BACs using different Tn-10 transposons [7-12]. The transposon plasmid DNAs from pTnloxP-iTol2kan and pTnlox511-iTol2kan were each separately introduced into the BAC clones made competent using the calcium chloride procedure. Transformed colonies were selected on LB agar plates containing both chloramphenicol and kanamycin. Approximately a thousand transformed colonies were pooled [13], and low density cultures containing chloramphenicol, kanamycin and ampicillin were set up. The cultures were induced with IPTG at early log phase of growth, and incubated further for three hours. Cells were spun down, re-suspended in one-tenth volume of fresh LB in the absence of antibiotics, and infected with phage P1. Two hours into the infection, cells were treated with chloroform and lysed, the supernatant isolated and used to infect fresh NS3516 cells. These were then plated on LB agar plates containing both chloramphenicol and kanamycin. Deletion libraries generated with pTnlox511-iTol2kan gave better yields of un-catenated BACs if recovered from the Cre-expressing strain NS3529, instead of the Cre nonexpressing strain NS3516 (PKC unpublished observations). Several thousand member BAC deletion libraries were routinely obtained using this procedure with both pTnloxP-iTol2kan and pTnlox511-iTol2kan.

\section{Analysis of BAC DNA from deletion libraries}

DNA from BAC clones was isolated and analyzed by Field Inversion Gel Electrophoresis (FIGE) after Not I digestion as previously described [14]. The Not I sites at either end of the original BAC are deleted upon lox-Cre recombination. The Not I sites in the transposon plasmids pTnloxP-iTol2kan and pTnlox511-iTol2kan are designed such that the BAC-vector DNA band is altered in size for authentic lox-Cre recombinants $[8,9]$. Thus deletions with pTnloxP-iTol2kan result in shortening of the BAC-vector DNA band, while those with pTnlox511-iTol2kan become devoid of the vector band (see reference [15], and the web site http://bacpac.chori. org/ptarbac21.htm for details of pTARBAC2.1 vector used for the zebrafish BAC library). As a result lox-Cre independent deletions, and/or rearrangements, occurring within the genomic insert are easily distinguished from authentic lox-Cre recombinations originating from an end of insert DNA [8-12].

To identify the newly created end, BAC deletions of the desirable size were then sequenced with primers 
based at the ends of the Tn10 transposons retained after deletion formation, for example pink end $\mathrm{R}$ and green end $\mathrm{L}$ in deletions made with pTnloxP-iTol2kan and pTnlox511-iTol2kan, respectively (see Figure 1 and reference 8). Orientation of the iTol2kan cassette in $\mathrm{BAC}$ clones was determined by direct sequencing of the BAC DNA with primers located at the ends of the kanamycin gene and going outwards. These are: Tkan1: $\mathrm{d}$ (CTGCGTGCAATCCATCTTGTTC) and Tkan2: d (CCTTCTTGACGAGTTCTTCTG)

\section{Injection of BAC DNA into zebrafish embryos}

DNA from chosen BAC clones were purified using the Qiagen tip procedure described earlier [7,14], and injected into zebrafish eggs as described earlier [4,9]. BACs functionalized with iTol2kan were typically injected with a mixture of 30-50 ng/uL BAC DNA plus 25-50 ng/uL transposase mRNA into one-cell stage zebrafish embryos using previously described procedures [4].

Procedures \& Primers for the "excision assay" in embryos transiently expressing EGFP

Excision assay was performed following a previously described procedure [16]. Primers used for this analysis are: kan629-outFwd: d (TCTGGATTCATCGACTGTGG) and kan186-outRev: d (GTCCTGCAGTTCATTCAGGG)

\section{Analysis of transgenic zebrafish}

Microscopy: Developing embryos were analyzed for EGFP expression between 24 and 72 hours post fertilization, using a Nikon Diaphot Fluorescence microscope with a Nikon high pressure mercury lamp as the excitation source. Embryos were photographed with a RT Spot from Diagnostic Instruments, Michigan, USA.

\section{Results}

We have developed novel methodology to introduce the repeat ends of Tol2 in an inverted orientation (iTol2) into BACs with high efficiency. The iTol2 DNA cassettes are placed precisely at the ends of the genomic DNA insert in BACs, while simultaneously trimming that insert DNA end by Cre-lox recombination. The end of lesser interest of the insert DNA is therefore chosen to introduce iTol2. In addition to its ease of manipulation, a key advantage of the procedure is the generation of a large collection of BACs with iTol2 introduced at the chosen end of the genomic DNA insert.

\section{Construction of transposon plasmids pTnloxP-iTol2kan and pTnlox511-iTol2kan}

The DNA cassette, containing the $200 \mathrm{bp}$ and $150 \mathrm{bp}$ repeat ends of the vertebrate transposon Tol2 placed in the inverted orientation and flanking the kanamycin resistance gene (iTol2kan), was amplified by PCR. The sequence recognized by the restriction enzyme Asc I was built into the 5 ' end of each primer, as indicated in Methods. After sub-cloning in pCR2.1 vector, the amplified DNA was excised and ligated at the unique Asc I site of plasmids pTnMarkerless 2 and pTnlox511(B)markerless 1 that were described previously $[11,8]$. The two iTol2 transposon plasmids, each containing both orientations of the iTol2kan cassette at the Asc I site, are shown in Figure 1. Deletion libraries of BACs were generated with a mixture containing both these orientations

\section{Tn10 plasmids with iTol2kan}

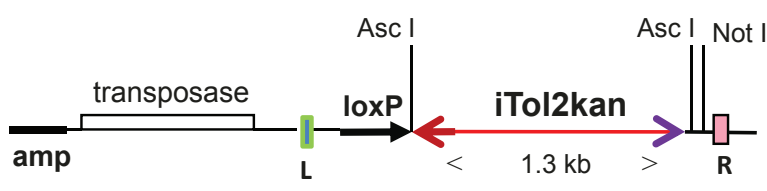

pTnloxP-iTol2kan

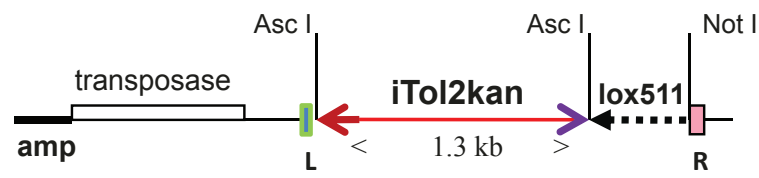

pTn/ox511-iTol2kan

Figure 1 Schematic of pTnloxP-iTol2kan and pTnlox511-iTol2kan: The iTol2kan DNA cassette was inserted at the Asc I site in both pTnMarkerless2 and pTnlox511(B)markerless1 plasmids, in either orientation. Mixtures of plasmids containing both orientations of iTol2 kan in pTnMarkerless2, and pTnlox511(B)markerless1, were used for generating deletion libraries in BACs. Clones from the library represented both orientations of the iTol2kan in them. The vertical boxes marked R (pink) and L (green), adapted from reference [8], indicate the 70 bp inverted repeat ends of the $\mathrm{Tn} 10$ transposon. The genes coding for transposase and ampicillin (amp) resistance are located outside the 70 bp inverted repeat ends. The arrows, thick black and broken and not drawn to scale, represent sequences for loxP and lox511, respectively. 
of iTol2kan for either the loxP or lox511 Tn10 plasmids. Note that the iTol2kan cassette is placed in front of the loxP or lox511 sites in the two transposons. Thus iTol2kan would be retained in the BAC DNA after lox-Cre recombination between the transposed and corresponding endogenous lox-sites at either end of BAC genomic inserts. We have previously demonstrated that crossrecombination between loxP and lox511 sites using phage-P1 generated Cre protein during a normal phage infection is negligible [8].

\section{Introducing iTol2kan at the lox511 end of insert DNA in BACs}

Progressive deletions of genomic DNA in BACs from either the loxP or lox511 end have been described $[7,8]$. An important feature of the technology is the simplicity of determining exactly where in the BAC DNA the loxPor lox511-transposon inserted to create the truncation $[7,8]$. Another significant feature is the ability to precisely introduce reporter genes and other DNA cassettes at the newly created end in the large BAC clone. The sequence in front of the loxP or lox511 arrowhead as shown in Figures 2 and 3 is retained after the recombination event that creates the deletion (see also reference [9]). The arrow orientation refers to the directionality of the loxP or lox511 sequences. This particular feature is used here to place the repeat ends of the vertebrate transposon Tol2 in the inverted orientation, and flanking a kanamycin resistance gene, at either the loxP or lox511 end of the BAC DNA.

Two types of BACs from the zebrafish library were used to demonstrate the efficacy of this method: BACs containing the Amyloid Precursor Protein gene of zebrafish (APPb) functionalized with EGFP enhancer-traps as described in an earlier study [9], and a BAC containing the fgf 24 gene functionalized with the EGFP gene by galK-mediated homologous recombination $[4,5]$. These are shown schematically in Figures 2 and 3, respectively.

Thus a $104 \mathrm{~kb}$ APPb-BAC clone with a $97 \mathrm{~kb}$ genomic insert DNA, containing the basal promoter EGFP gene regulated by $\sim 94 \mathrm{~kb}$ upstream DNA of the $\mathrm{APPb}$ gene, was chosen for insertion of iTol2kan at the lox511 end (Figure 2). When injected into zebrafish embryos, DNA from this and similar clones expressed GFP fluorescence exclusively in neurons [9]. Plasmid DNA from pTnlox511iTol2kan was introduced into this BAC clone using calcium chloride transformation as previously described [12]. A deletion library comprising of at least 3000 clones was isolated from the iTol2kan transposon plasmid transformed BAC culture using procedures described previously [12]. DNA was isolated from clones of this library and analyzed by FIGE. Arrays of BAC deletions, each containing the iTol2kan at the lox511 end and the EGFP gene at the loxP end of genomic insert DNA, are shown in Figure 4A. Note that Cre recombination of lox511 sites, one endogenous to BAC vector and the other transposed by Tnlox511-iTol2kan, deletes the Not I site existing at the lox511 end of the starting BAC DNA. Consequently a separate Not I-Not I vector DNA band is not present in deletion clone DNA in the FIGE gels of panels A and B of Figure 4. Clone DNAs shown in FIGE Panels A and B were obtained from libraries made with different $\mathrm{APPb}$ BACs that contained the EGFP enhancer-trap located either $2.5 \mathrm{~kb}$ upstream (Panel A), or $4 \mathrm{~kb}$ upstream (Panel $\mathrm{B}$ ) of the transcription start site of the APPb gene (see ref. [9] for details). Both orientations of the iTol2kan cassette were found in clones in the FIGE arrays shown in panels $A$ and $B$ of Figure 4. This was determined by direct sequencing of the BAC DNA using primers, Tkan 1 or Tkan2, located at the ends of the kanamycin gene in the iTol2kan cassette and going outwards. In approximately half the clones, sequencing with either Tkan1 or Tkan2 primer helped determine the location of the newly created end in the APPb BAC made by pTnlox511-iTol2kan in the $\mathrm{APPb}$ gene region of chromosome 9 of zebrafish. BLAST analysis was used to identify these locations (shown in Additional file 1), which were consistent with the size of the BACs estimated from the markers in the FIGE arrays shown in Figure 4 Panels A and B.

\section{Introducing iTol2kan at the loxP end of insert DNA in BACs}

The APPb BACs functionalized with EGFP enhancertraps at the loxP end of genomic insert could not be used to introduce iTol2 cassettes again at the loxP end. Using the loxP-iTol2kan transposon on these BACs would only delete the EGFP enhancer-trap reporter cassette. Therefore a zebrafish BAC clone functionalized with the EGFP gene by galK-mediated homologous recombination was selected to introduce iTol2kan at the loxp end (shown schematically in Figure 3). The open reading frame of EGFP gene was fused to the initiator codon of the fgf 24 gene in this BAC. The transposon plasmid pTnloxPiTol2kan, shown schematically in Figure 1, was introduced into the fgf24:EGFP BAC using the calcium chloride transformation procedure. Note that the fgf24:EGFP BAC DNA was isolated from the host SW 102, and reintroduced by electroporation into DH10B cells to avoid potential complications arising from the temperature sensitive $\lambda$-prophage in the previous host (see Methods).

A deletion library of several thousand members was generated with fgf24:EGFP BAC using pTnloxP-iTol2kan, as described earlier [12]. Analysis of deletion clone DNA by FIGE after Not I digestion is shown in panel $C$ of Figure 4. Because the EGFP cassette was introduced at fgf 24 


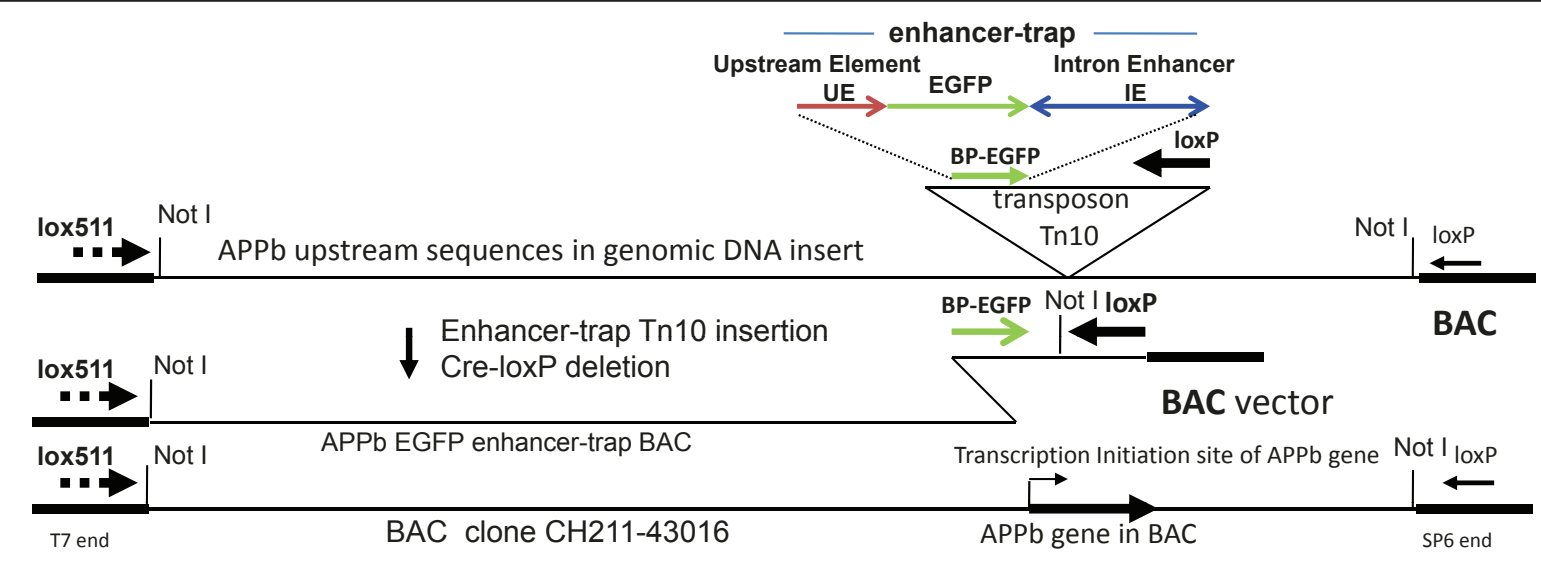

Inserting Tn/ox511-iTol2kan in APPb EGFP enhancer-trap BAC

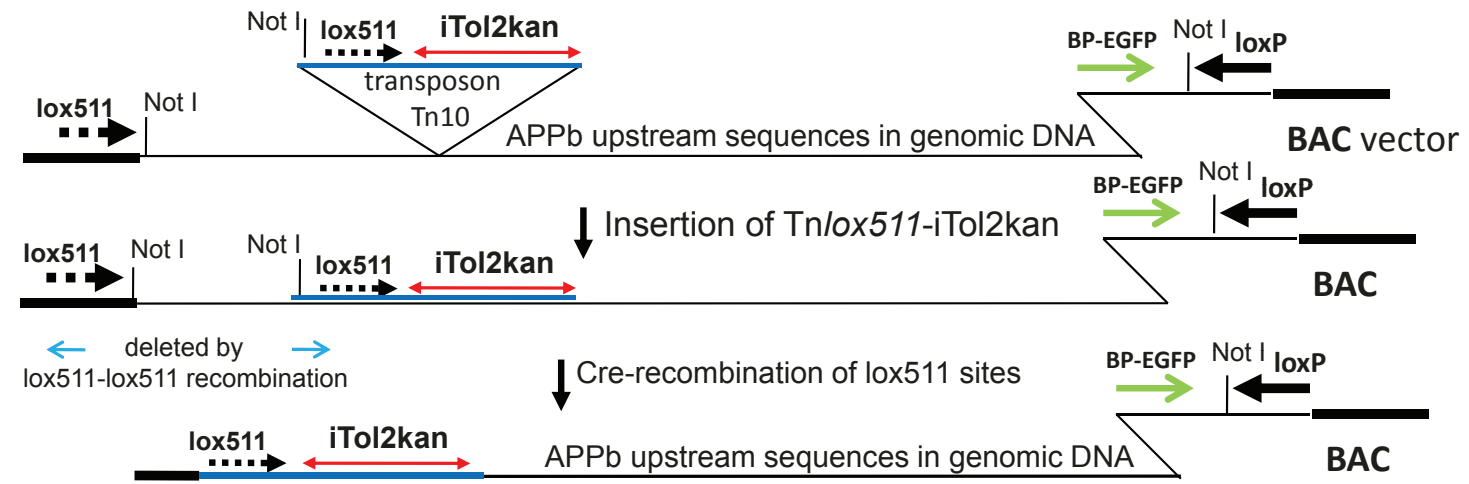

Figure 2 Schematic of deletion formation in APPb:EGFP-enhancer-trap BAC by pTn/ox511-iTol2kan: Starting BAC APPb EGFP enhancertrap was generated by inserting the enhancer-trap transposon (inverted triangle) is shown, along with the location of the APPb gene within the BAC clone (adapted from reference [9]). The insertion of the Tnlox511-iTol2kan transposon, and the resulting lox511-lox511 deletion mediated by Cre protein are shown in the lower half.

translation start site, it was important to isolate clones with genomic insert DNA longer than $75 \mathrm{~kb}$ (see Figure $3)$. Therefore only clones that fulfill this criterion are shown in the FIGE of Figure 4C.

The fgf24:EGFP BAC deletions shown in panel $C$ were sequenced with transposon based primer, Seq 1 [7], to determine the location of the newly created end containing the iTol2kan cassette on the fgf 24 BAC DNA. BLAST analysis of sequences helped determine their locations on a chromosomal map (see Additional file 1). These were consistent with the size of clone DNA in Figure 4, panel C.

\section{Expression analysis of iTol2-containing APPb:EGFP BAC} and fgf24:EGFP BAC in zebrafish embryos

Deletion clone DNAs marked with the blue arrowheads, in Panels A, B and C of Figure 4, were further purified using the Qiagen-tip procedure $[7,14]$, and injected into zebrafish eggs for expression and/or "excision assay" analyses along with mRNA capable of expressing Tol 2 transposase [1-4].
Excision Assay analyses to confirm authentic recognition of iTol2-ends by Tol2 -transposase

Recognition and processing of the BAC DNA, containing the iTol2kan cassette, by the Tol2 transposase enzyme upon entry into zebrafish oocytes can be evaluated using the "excision assay" [16]. Thus the embryos injected with DNA from APPb:EGFP BAC and fgf24: EGFP BAC were analyzed using the "excision assay". The results shown in the lower panels of Figure 5 clearly indicate that all of the BAC DNAs tested were processed by the Tol 2 transposase. Transient expression patterns of embryos analyzed with the excision assay are shown in panels A-D of Figure 5. The results demonstrate that iTol2kan at lox511-end for BACs 2 and 13, and the loxP-end for clones 35 and 37, respectively, are recognized accurately by the Tol 2 transposase. Note that BACs 2, 35 and 37 are larger than $100 \mathrm{~kb}$.

\section{Germline expressions of iTol2 containing BACs}

Transgenic zebrafish lines were established from injections of EGFP BAC DNAs shown in lanes 15, 27 and 37 


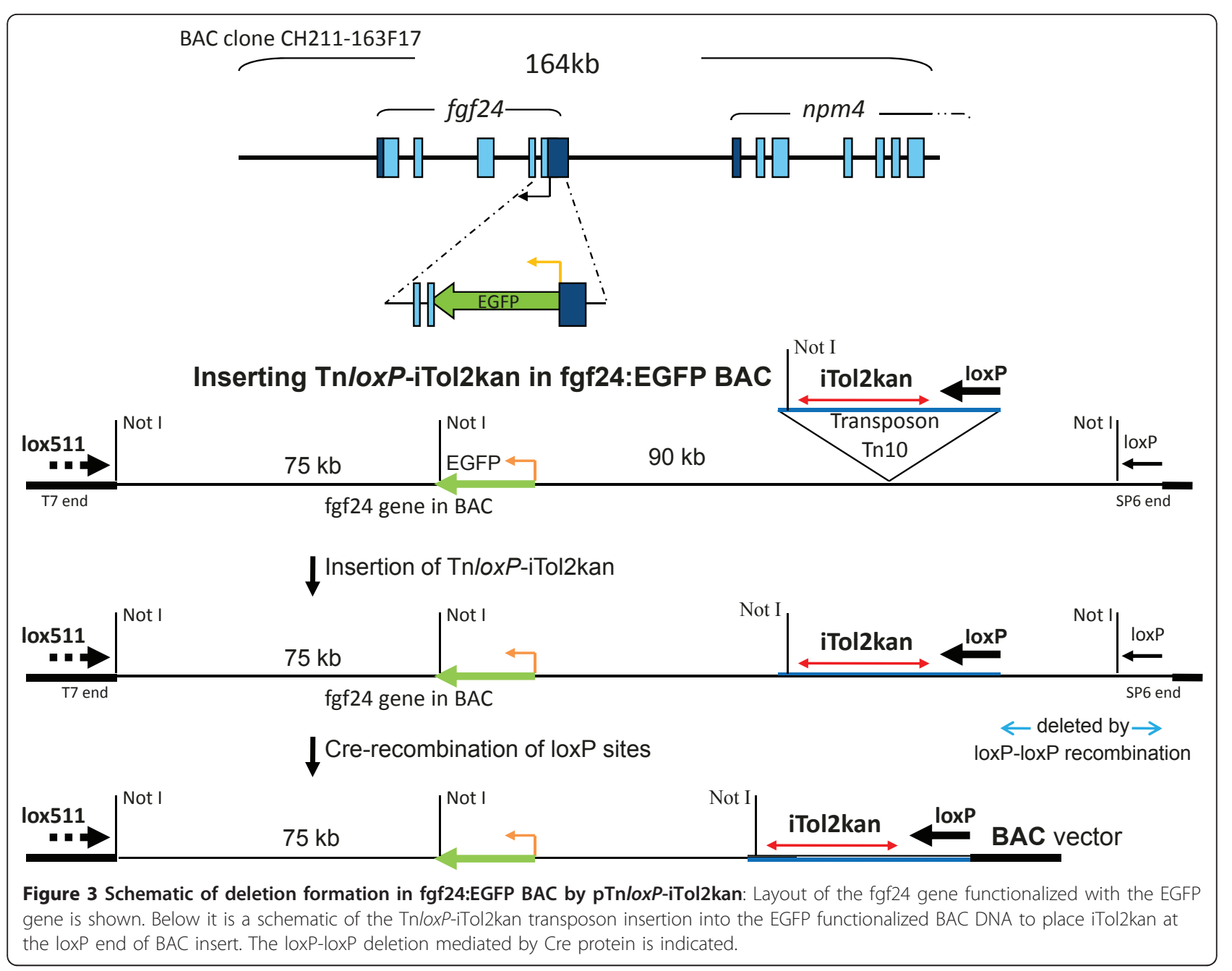

in FIGE panels A, B and C of Figure 4, respectively. The EGFP fluorescence of the F1 zebrafish lines are shown in Figure 6, panels A-C, for BAC clones 15, 27 and 37 respectively. Expression of EGFP fluorescence is restricted to neurons and highly specific to the hind brain and spinal cord in F1 transgenic zebrafish created from injections of iTol2-APPb:EGFP BACs 15 and 27, as seen in panels A and B of Figure 6. The slight difference in the expression patterns observed in the two panels might be due to the different APPb BACs used; clones 15 and 27 have DNA extending till $2.5 \mathrm{~kb}$ and 4 $\mathrm{kb}$ upstream of the $\mathrm{APPb}$ transcription start site, respectively.

Germline expression of iTol2 retrofitted fgf24:EGFP BAC clone 37 is shown in Figure 6C. F1 embryos from injections with fgf24:EGFP BAC 37 DNA showed GFP expression patterns almost identical to those from injections with full length fgf24:EGFP BAC DNA that had the iTol2 cassette introduced by GalK mediated recombination (5, Gembu Abe, Koichi Kawakami unpublished observations). Taken together, the results indicate that BACs larger than $100 \mathrm{~kb}$ are not only recognized and processed effectively by the Tol2 transposase, but are also integrated efficiently into the zebrafish chromosome when iTol2 is introduced into the BAC DNA ends using a loxP or lox511 Tn10 transposon.

\section{Discussion}

We have developed a novel approach to rapidly introduce repeat ends of the vertebrate transposon Tol2, in an inverted orientation, at the ends of BAC DNA. The Tn10 transposons used for the purpose carry both iTol2 ends and lox sites that can be recombined by Cre protein with the corresponding lox sites endogenous to the BAC vector and flanking the genomic DNA insert. As the insertions of these iTol2-Tn10 transposons into genomic DNA in BACs appear to be largely random (see references $[10,13]$ for discussions), large collections of BACs with iTol2 at the newly trimmed end are generated in a single experiment. Such libraries are 


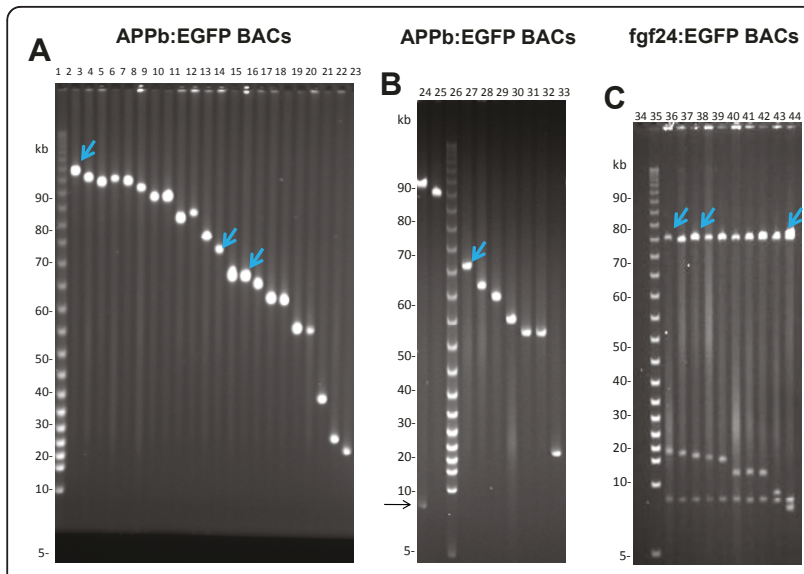

Figure 4 FIGE analysis of clones from libraries generated with insertion of either pTn/ox511-iTol2kan (panels A and B) or pTnloxP-iTol2kan (panel C). Deletion clone DNAs shown in FIGE Panels A and B were obtained from libraries made with different APPb BACs that contained the EGFP enhancer-trap located either $2.5 \mathrm{~kb}$ upstream (Panel A), or $4 \mathrm{~kb}$ upstream (Panel B) of the transcription start site of the APPb gene. Panel $\mathbf{C}$ shows clone DNA from the library generated with fgf24:EGFP using Tn/oxP-iTol2kan transposon. Only clones containing an intact $75 \mathrm{~kb}$ fragment are shown here. All clone DNAs were digested with Not I enzyme prior to FIGE analysis. The blue arrows indicate the BAC clones tested in zebrafish for expression and/or "excision assay" analyses. All clones are numbered according to the lanes in which their DNA appears, and are referred to as such throughout the text. Lanes 1, 26 and 34 are marker lanes containing the $5 \mathrm{~kb}$ ladder, and BAC 24 (in lane 24) is the starting APPb BAC used to generate the deletions in panel $B$. The arrow at the side and bottom of Panel $B$ indicates the position of the BAC vector DNA band, which in this case is $\sim 7 \mathrm{~kb}$. Size of DNA bands in $\mathrm{kb}$ is indicated on the side of each panel.

uniquely suited for functionally mapping long-range gene regulatory sequences using transgenic animals. Thus multiple BACs from a contig spanning a genetic locus should enable such functional analyses to be extended over large regions of the genome. The approach does not require selecting sequences for mutational analyses to test their gene regulatory potential, and is therefore unbiased. It enables enhancer-trap containing BACs, or BACs retrofitted with EGFP cassettes using sequence homology based recombination, to be readily converted into deletion libraries with integrated iTol2 ready for chromosome integration. Compared to traditional approaches for enhancer-trapping used with whole genomes in animals (see references [17-25]), our approach using enhancer-traps in individual BACs has the potential to allow a more uniform coverage of the genome because the baseline efficiency of trap insertion is reset for individual BACs in the bacterial host. Although there appear to be vast regions of the genome refractory to enhancer-trapping by traditional means, to date we have not encountered BACs refractory to Tn10 transposon insertions.

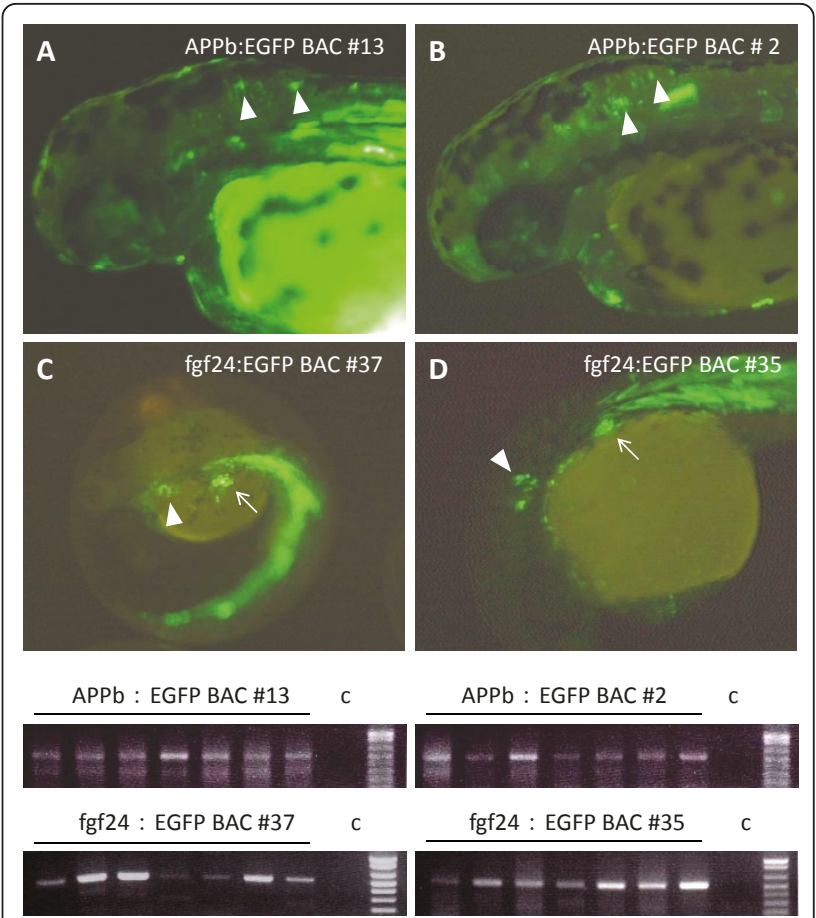

Figure 5 "Excision assay" performed with genomic DNA isolated from 2-day old zebrafish embryos: GFP expression in the embryos injected with iTol2-BACs were observed between 28 and 48 hours post fertilization (hpf) (upper panels). APPb:EGFP BAC 13 (panel 5A), APPb:EGFP BAC 2 (panel 5B), fgf24:EGFP BAC 37 (panel 5C) and fgf24:EGFP BAC 35 (panel 5D) display green fluorescence. The white arrowheads indicate the expected tissuespecific expressions of GFP in these embryos. The iTol2-BAC injected embryos were analyzed using the "excision assay" (lower panels) $[16,4]$.

The iTol2 insertions into BAC DNA are performed using Tn10 transposons carrying loxP or lox511 sites, and do not rely upon sequences existing in the genomic inserts of BACs. Consequently, the Tn10 transposons developed here are applicable to all BACs in the public domain. This feature is unlike the targeting vectors used in methods based on recombining homologous sequences, which need to be constructed anew for each BAC. The use of radioactive isotopes is also prevented, as Southern blotting is not required in retrofitting BACs using Tn10 transposons.

Varying degrees of promiscuity in recombining different mutant lox sites, including the lox511 mutant with wild type loxP, using both partially purified Cre-extracts in vitro [26-29], and Cre over-expressed in cells [30-32] have been reported. For example, cross recombination between loxP and lox511 has been reported to occur at efficiencies ranging from 5 to $100 \%$ under those experimental conditions that express Cre constitutively $[26,27,29-32]$. We have not observed such cross-recombination. High levels of stringency in vivo in recombining 

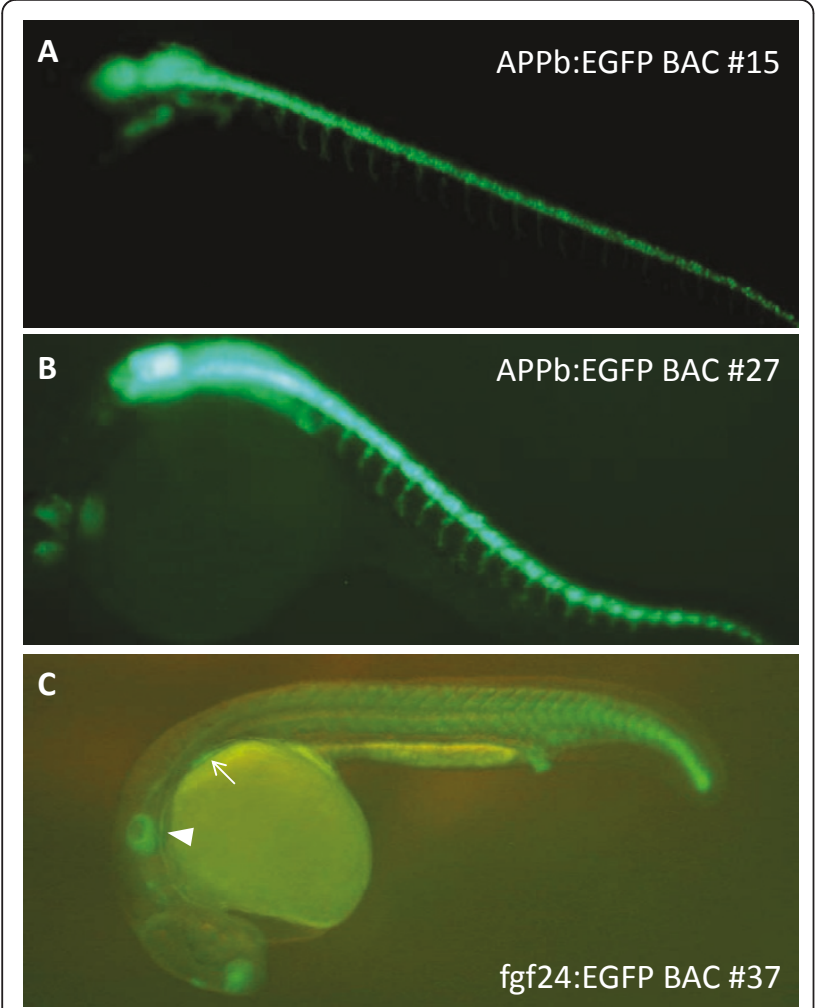

Figure 6 EGFP fluorescence in transgenic F1 zebrafish: Panel A Germline expression of EGFP fluorescence in zebrafish embryos with DNA from APPb:EGFP BAC clone 15. Panel B shows the germline expression of EGFP fluorescence with DNA from APPb:EGFP BAC 27, and $\underline{P a n e l} \mathbf{C}$ shows germline expression from fgf24:EGFP BAC clone 37.

identical lox sites [8,33], or lox sites with at least identical spacers [34], have been achieved with Cre protein expressed from its native source, namely a phage P1 infection $[8,33,34]$. Depending on whether a loxP or a lox511 transposon is used, truncations from the corresponding lox- end of insert DNA in BACs can be made readily and exclusively with high stringency. Thus insertions of iTol2 with the resulting truncations of genomic DNA from either end are not only efficient, but also highly specific to that end.

A notable drawback of the transposon based approach appears to stem from the very feature that makes it so efficient: the P1 headful packaging strategy used to isolate the functionalized BAC so easily also limits the size of the BAC clone that can be analyzed to $\sim 110 \mathrm{~kb}$. Although the genomic DNA insert is truncated in the process to a size of $\sim 103 \mathrm{~kb}$, the remainder BAC vector, it is unlikely to be a disadvantage in most applications because a majority of vertebrate genes can be housed in their entirety within this size limit. We note that almost half of evolutionarily conserved non-coding gene-regulatory sequences in vertebrate genomes [35], and probably a similar fraction of those that are conserved in function and shape but not in sequence [36,37], are located within this span of DNA adjoining transcription start sites of genes.

It is clear that the transposon retrofitting strategy and those based on homologous recombination have strengths that appear somewhat complimentary in nature. Therefore a judicious approach might be to use a combination of the two methodologies, as demonstrated here for the fgf24:EGFP BAC and in an earlier study [38]. The procedures described here should allow one to readily generate libraries of EGFP functionalized BACs, progressively truncated from an end and carrying the iTol2 cassette, ready for integration into chromosomes of at least fish and mice [4].

\section{Conclusion}

The repeat ends of the Tol2 transposon in the inverted orientation, and flanking a kanamycin resistance gene (iTol2), was introduced by a novel procedure to the ends of BAC DNA. We show that these iTol2 inserted BACs are efficiently recognized by the Tol 2 transposase, and successfully integrate into the germline of zebrafish. Compared to previous methods using recombination of homologous sequences to introduce iTol2 ends into BACs, our lox-Cre recombination approach rapidly generates, in a single experiment, a large collection of BACs with iTol2 placed at an end that is also progressively truncated. The libraries of iTol2 inserted BACs generated should facilitate integration of trimmed single genes into the germline, and help functionally map cisacting gene regulatory sequences in animals. The approach should be applicable to a wider variety of BACs, including those with sequence repeats.

\section{Additional material}

Additional file 1: Location of iTol2kan insertions (new BAC ends) in APPb:EGFP and fgf24:EGFP BACs. Newly created ends containing the iTol2kan cassette were sequenced with primers Tkan1 and Tkan2 for APPb:EGFP BAC deletions (Panel A), and with primer Seq1 for fgf24:EGFP BAC deletions (Panel B). The sequences were BLASTed to the zebrafish genome, and location of the new ends of BACs where the iTol2kan cassettes are placed indicated in panels $\mathbf{A}$ and $\mathbf{B}$. Clone numbers are as in lanes shown by the blue arrowheads in Figure 4.

List of abbreviations

BAC: bacterial artificial chromosome; FIGE: Field inversion gel electrophoresis; APPb: amyloid precursor protein gene b; EGFP:enhanced green fluorescent protein.

\section{Acknowledgements}

The project described was supported by Award Number P20MD000175 from the National Center On Minority Health And Health Disparities. The content is solely the responsibility of the authors and does not necessarily represent the official views of the National Center On Minority Health And Health 
Disparities or the National Institutes of Health, and funds from the North Carolina Biotechnology Center. We thank Shanta Mackinnon and Charles Hatcher for zebrafish eggs, and Ms Rosalind Grays, Connie Keys, Crystal McMichael and Darlene Laws for support and encouragement. We thank Dr. Derek Norford for valuable suggestions on the manuscript, and MaryByrd Ewell, Hayden Abene and Christopher Hayes for help with initial screening of colonies. PKC would like to thank Drs. Ken Harewood and Sean Kimbro for encouragement and funding support.

\section{Author details}

'Julius L. Chambers Biomedical/Biotechnology Research Institute \& Department of Chemistry, North Carolina Central University, 1801 Fayetteville Street, Durham, NC 27707, USA. ${ }^{2}$ Division of Molecular and Developmental Biology, National Institute of Genetics, Mishima, Shizuoka 411-8540, Japan. ${ }^{3}$ Department of Chemistry, University of North Carolina at Chapel-Hill, Chapel-Hill, NC 27599. USA.

\section{Authors' contributions}

LAS screened iTol2 inserted BACs by FIGE, sequenced iTol2 BAC ends, injected BAC DNA into zebrafish embryos, and documented positive embryos using photo-microscopy. GA injected iTol2 BACs into zebrafish embryos, performed excision assays, analyzed embryos positive for expression and contributed to writing the manuscript. MAE screened libraries of iTol2 BACs, isolated clones of desirable size using FIGE and injected BAC DNA into zebrafish eggs. HMW screened and analyzed the small plasmids carrying iTol2kan and lox sites, and helped write the manuscript. PKC designed the study, helped with design and construction of the transposon plasmids, generated the iTol2 libraries of BAC deletions, purified BAC DNA for injections using Qiagen columns, and was responsible for writing the article with KK. Both KK and PKC critically evaluated the study. All authors read and approved the final manuscript.

Received: 26 April 2011 Accepted: 7 July 2011 Published: 7 July 2011

\section{References}

1. Kawakami K, Shima A, Kawakami N: Identification of a functional transposase of the Tol2 element, an Ac-like element from the Japanese medaka fish, and its transposition in the zebrafish germ lineage. Proc Natl Acad Sci USA 2000, 97:11403-11408.

2. Kawakami K, Takeda H, Kawakami N, Kobayashi M, Matsuda N, Mishina M: A transposon-mediated gene trap approach identifies developmentally regulated genes in zebrafish. Developmental Cell 2004, 7:133-144

3. Urasaki A, Morvan G, Kawakami K: Functional dissection of the Tol2 Transposable Element Identified the Minimal cis- Sequence and a Highly Repetitive Sequence in the Subterminal Region Essential for Transposition. Genetics 2006, 174:639-649.

4. Suster ML, Sumiyama K, Kawakami K: Transposon-mediated BAC transgenesis in zebrafish and mice. BMC Genomics 2009, 10:477.

5. Warming S, Costantino N, Court DL, Jenkins NA, Copeland NG: Simple and highly efficient BAC recombineering using galK selection. Nucleic Acids Res 2005, 33:e36.

6. Chatterjee PK, Coren JC: Isolating large nested deletions in bacterial and P1 artificial chromosomes by in vivo P1 packaging of products of Crecatalyzed recombination between the endogenous and a transposed loxP site. Nuc Acids Res 1997, 25:2205-2212.

7. Chatterjee PK, Yarnall DP, Haneline SA, Godlevski MM, Thornber SJ, Robinson PS, Davies HE, White NJ, Riley JH, Shepherd NS: Direct Sequencing of Bacterial and P1 Artificial Chromosome Nested-deletions for Identifying Position-Specific Single Nucleotide Polymorphisms. Proc Natl Acad Sci (USA) 1999, 96:13276-13281.

8. Shakes LA, Garland DM, Srivastava DK, Harewood KR, Chatterjee PK: Minimal Cross-recombination between wild type and loxP511 sites in vivo facilitates Truncating Both Ends of Large DNA Inserts in pBACe3.6 and Related Vectors. Nucleic Acids Research 2005, 33:e118.

9. Shakes LA, Malcolm TL, Allen KL, De S, Harewood KR, Chatterjee PK: Context dependent function of APPb Enhancer identified using Enhancer Trap-containing BACs as Transgenes in Zebrafish. Nucleic Acids Research 2008, 36:6237-6248.

10. Gilmore RC, Baker J Jr, Dempsey S, Marchan R, Corprew RNL Jr, Maeda N, Smithies O, Byrd G, Bukoski RD, Harewood KR, Chatterjee PK: Using PAC nested-deletions to order contigs and microsatellite markers at the high repetitive sequence containing Npr3 gene locus. Gene 2001, 275:65-72.

11. Chatterjee PK, Mukherjee S, Shakes LA, Wilson W III, Harewood KR, Byrd G: Selecting Transpositions of a Markerless Transposon Using Phage P1 Headful Packaging: New Transposons for Functionally Mapping Long Range Regulatory Sequences in BACs. Analytical Biochemistry 2004, 335:305-315.

12. Chatterjee PK: Retrofitting BACs and PACs with LoxP Transposons to Generate Nested Deletions. "Bacterial Artificial Chromosomes". In Methods in Mol. Biology series vol 255. Volume 1. Edited by: Shaying Zhao, Marvin Stodolsky. The Humana Press Inc; 2004:231-241.

13. Chatterjee PK, Briley LP: Analysis of a Clonal Selection Event during Transposon-Mediated Nested-Deletion Formation in Rare BAC and PAC Clones. Analytical Biochemistry 2000, 285:121-126.

14. Chatterjee PK, Baker JC Jr: Preparing Nested Deletions Template DNA for Field Inversion Gel Electrophoresis Analyses and Position-Specific End Sequencing With Transposon Primers. "Bacterial Artificial Chromosomes". In Methods in Mol. Biology series vol 255. Volume 1. Edited by: Shaying Zhao, Marvin Stodolsky. The Humana Press Inc; 2004:243-254.

15. Osoegawa K, Woon PY, Zhao B, Frengen E, Tateno M, Catanese JJ, de Jong PJ: An improved approach for construction of bacterial artificial chromosome libraries. Genomics 1998, 52:1-8.

16. Kawakami K, Shima A: Identification of the Tol2 transposase of the medaka fish Oryzias latipes that catalyzes excision of a non-autonomous Tol2 element in zebrafish Danio rerio. Gene 1999, 240:239-244.

17. O'Kane CJ, Gehring WJ: Detection in situ of genomic regulatory elements in Drosophila. Proc Natl Acad Sci USA 1987, 84:9123-9127.

18. Wilson C, Pearson RK, Bellen HJ, O'Kane CJ, Grossniklaus U, Gehring WJ: Pelement-mediated enhancer detection: an efficient method for isolating and characterizing developmentally regulated genes in Drosophila. Genes Dev 1989, 3:1301-1313.

19. Korn R, Schoor M, Neuhaus H, Henseling U, Soininen R, Zachgo J, Gossler A: Enhancer trap integrations in mouse embryonic stem cells give rise to staining patterns in chimaeric embryos with a high frequency and detect endogenous genes. Mech Dev 1992, 39:95-109.

20. Grabher C, Henrich T, Sasado T, Arenz A, Wittbrodt J, Furutani-Seiki M: Transposon-mediated enhancer trapping in medaka. Gene 2003, 322:57-66.

21. Balciunas D, Davidson AE, Sivasubbu S, Hermanson SB, Welle Z, Ekker SC: Enhancer trapping in zebrafish using the Sleeping Beauty transposon. BMC Genomics 2004, 5:62.

22. Kawakami K, Takeda H, Kawakami N, Kobayashi M, Matsuda N, Mishina M: A transposon-mediated gene trap approach identifies developmentally regulated genes in zebrafish. Developmental Cell 2004, 7:133-144.

23. Kawakami K: Transposon tools and methods in zebrafish. Developmental Dynamics 2005, 234:244-254.

24. Ellingsen S, Laplante MA, Konig M, Kikuta $H$, Furmanek T, Hoivik EA, Becker TS: Large-scale enhancer detection in the zebrafish genome. Development 2005, 132:3799-3811.

25. Nagayoshi S, Hayashi E, Abe G, Osato N, Asakawa K, Urasaki A, Horikawa K, Ikeo K, Takeda H, Kawakami K: Insertional mutagenesis by the Tol2 transposon-mediated enhancer trap approach generated mutations in two developmental genes: tcf7 and synembryn-like. Development 2008, 135(1):159-169.

26. Hoess RH, Wierzbicki A, Abremski K: The role of the loxP spacer region in P1 site-specific recombination. Nucleic Acids Res 1986, 14:2287-2300.

27. Lee $G$, Saito I: Role of nucleotide sequences of loxP spacer region in Cremediated recombination. Gene 1998, 216:55-65.

28. Langer SJ, Ghafoori AP, Byrd M, Leinwand L: A genetic screen identifies novel non-compatible loxP sites. Nucleic Acids Res 2002, 30:3067-3077.

29. Missirlis PI, Smailus DE, Holt RA: A high-throughput screen identifying sequence and promiscuity characteristics of the loxP spacer region in Cre-mediated recombination. BMC Genomics 2006, 7:73-85.

30. Sheren J, Langer SJ, Leinwand LA: A randomized library approach to identifying functional lox site domains for the Cre recombinase. Nucleic Acids Res 2007, 35:5464-5473.

31. Siegel RW, Jain R, Bradbury A: Using an in vivo phagemid system to identify non-compatible loxP sequences. FEBS lett 2001, 505:467-473.

32. Wang Z, Engler P, Longacre A, Storb U: An efficient method for highfidelity $B A C / P A C$ retrofitting with a selectable marker for mammalian cell transfection. Genome Res 2001, 11:137-142. 
33. Chatterjee PK, Shakes LA, Srivastava DK, Garland DM, Harewood KR, Moore KJ, Coren JS: Mutually Exclusive Recombination of Wild Type and Mutant loxP Sites in vivo Facilitates Transposon-Mediated Deletions from Both Ends of Genomic DNA in PACs. Nucleic Acids Research 2004, 32:5668-5676.

34. Chatterjee PK, Shakes LA, Stennett N, Richardson VL, Malcolm TL, Harewood KR: Replacing the wild type loxP site in BACs from the public domain with lox66 using a lox66 transposon. BMC Res Notes 2010, 3:38.

35. Woolfe A, Goodson M, Goode DK, Snell P, McEwen GK, Vavouri T, Smith SF, North P, Callaway H, Kelly K, Walter K, Abnizova I, Gilks W, Edwards YJ, Cooke JE, Elgar G: Highly conserved non-coding sequences are associated with vertebrate development. PLoS Biol 2005, 3:e7.

36. Fisher S, Grice EA, Vinton RM, Bessling SL, McCallion AS: Conservation of RET regulatory function from human to zebrafish without sequence similarity. Science 2006, 14:276-279.

37. Parker SC, Hansen L, Abaan HO, Tullius TD, Margulies EH: Local DNA topography correlates with functional noncoding regions of the human genome. Science 2009, 324(5925):389-92, Epub 2009 Mar 12.

38. Chi X, Chatterjee PK, Wilson W III, Zhang S-X, DeMayo F, Schwartz RJ: Complex Cardiac Nkx2-5 Gene Expression Activated By Noggin Sensitive Enhancers Followed By Chamber Specific Modules. Proc Natl Acad Sci (USA) 2005, 102:13490-13495.

doi:10.1186/1471-2164-12-351

Cite this article as: Shakes et al:: Generating libraries of iTol2-end insertions at BAC ends using loxP and lox511 Tn10 transposons. BMC Genomics 2011 12:351.

\section{Submit your next manuscript to BioMed Central and take full advantage of:}

- Convenient online submission

- Thorough peer review

- No space constraints or color figure charges

- Immediate publication on acceptance

- Inclusion in PubMed, CAS, Scopus and Google Scholar

- Research which is freely available for redistribution

Submit your manuscript at www.biomedcentral.com/submit 